\title{
Bulk viscosity in superfluid neutron star cores
}

\section{Modified Urca processes in $n p e \mu$ matter}

\author{
P. Haensel ${ }^{1}$, K. P. Levenfish ${ }^{2}$, and D. G. Yakovlev ${ }^{2}$ \\ 1 N. Copernicus Astronomical Center, Bartycka 18, 00-716 Warszawa, Poland \\ 2 Ioffe Physical Technical Institute, Politekhnicheskaya 26, 194021 St.-Petersburg, Russia
}

Received 22 December 2000 / Accepted 9 March 2001

\begin{abstract}
We study the bulk viscosity in neutron star cores due to modified Urca processes involving nucleons, electrons and muons and analyze its reduction by singlet-state or triplet-state superfluidity of nucleons. In combination with the results of our previous paper on the bulk viscosity due to direct Urca processes, a realistic description of the bulk viscosity in superfluid neutron star cores is obtained. Switching off direct Urca processes with decreasing density in a nonsuperfluid matter lowers the bulk viscosity by $3-5$ orders of magnitude. The presence of muons opens additional source of bulk viscosity due to muon Urca processes and lowers the threshold density of the electron direct Urca process. The superfluidity may strongly reduce the bulk viscosity and affect thus damping of neutron star vibrations.
\end{abstract}

Key words. stars: neutron - dense matter - bulk viscosity

\section{Introduction}

The bulk viscosity of neutron star matter determines damping of neutron star pulsations which could be excited during neutron star formation or triggered by instabilities developed during neutron star evolution (e.g., Cutler et al. 1990). The bulk viscosity can damp gravitational radiation driven instabilities in rotating neutron stars and therefore influence the maximum rotation frequency (e.g., Lindblom 1995; Zdunik 1996; Andersson \& Kokkotas 2000).

In this paper, we continue to study the bulk viscosity in neutron star cores (the layers of density $\rho \gtrsim$ $1.5 \times 10^{14} \mathrm{~g} \mathrm{~cm}^{-3}$ ) assuming that stellar matter consists of neutrons $n$, protons $p$, electrons $e$, and muons $\mu$ and taking into account superfluidity of nucleons. Protons and neutrons constitute strongly interacting Fermi liquids while electrons and muons constitute almost ideal, strongly degenerate gases. Electrons are ultrarelativistic; muons may be nonrelativistic or moderately relativistic, depending on density. The bulk viscosity in this matter is mainly produced by neutrino processes of Urca type. These processes are known to be divided into most powerful direct Urca processes and much weaker modified Urca processes.

A direct Urca process consists of two reactions, direct and inverse one,

$n \rightarrow p+l+\bar{\nu}_{l}, \quad p+l \rightarrow n+\nu_{l}$,

Send offprint requests to: P. Haensel, e-mail: haensel@camk.edu.pl where $l$ is either electron or muon and $\nu_{l}$ is an associated neutrino. Direct Urca processes are allowed (Lattimer et al. 1991) for some realistic equations of state at densities higher than certain threshold densities (of several $\rho_{0}$, where $\rho_{0}=2.8 \times 10^{14} \mathrm{~g} \mathrm{~cm}^{-3}$ is the standard nuclear matter density). Thus, they may be open in the inner cores of rather massive neutron stars producing large bulk viscosity. The threshold density for the muon process is always higher than for the electron one.

However, for many equations of state, direct Urca processes are forbidden by momentum conservation at any density in a neutron star core. Moreover, at $\rho \lesssim 3 \rho_{0}$ they are prohibited for the majority of equations of state. Thus, they do not operate in the low and medium-mass neutron stars and in the outer cores of almost all neutron star models. If so, the bulk viscosity is determined by the reactions of modified Urca processes

$n+N \rightarrow p+N+l+\bar{\nu}_{l}, \quad p+N+l \rightarrow n+N+\nu_{l}$,

where $N$ is an additional nucleon required to ensure momentum conservation. For instance, in the npe matter one has two modified Urca processes, with $N=n$ or $p$, which will be referred to as the neutron and proton branches of the modified Urca process (e.g., Friman \& Maxwell 1979; Yakovlev \& Levenfish 1995). In the npe $\mu$ matter, we have four processes corresponding to $N=n$ or $p$, and $l=e$ or $\mu$; they will be labeled as $N l$. The rates of the modified Urca processes are 3-5 orders of magnitude lower than those of the direct Urca processes. The modified Urca processes 
either have no density threshold (as the neutron branch in the npe matter) or have much lower thresholds than the direct Urca.

Sawyer (1989) and Haensel \& Schaeffer (1992) analyzed the bulk viscosity of non-superfluid npe matter produced by the neutron branch of the modified Urca process and by the nucleon direct Urca process, respectively.

Recently we (Haensel et al. 2000, hereafter Paper I) have considered the bulk viscosity due to the direct Urca processes in the npe $\mu$ matter with superfluid neutrons and protons. In the present paper we analyze the bulk viscosity of this matter produced by the modified Urca processes.

Let us remind that damping of stellar pulsations is produced also by shear viscosity (calculated for instance by Flowers \& Itoh 1976). Contrary to the bulk viscosity the shear viscosity grows with decreasing temperature $T$ and governs the damping of pulsations of cold neutron stars. Estimates show (e.g., Paper I) that the bulk viscosity determines the damping of pulsations in nonsuperfluid stars at $T \gtrsim 10^{8} \mathrm{~K}$ if direct Urca processes are open in the stellar cores or at $T \gtrsim 10^{9} \mathrm{~K}$ if direct Urca processes are forbidden.

\section{Bulk viscosity in non-superfluid matter}

General expression for the bulk viscosity $\zeta$ of nonsuperfluid npe $\mu$ matter produced by nonequilibrium (direct or modified) Urca processes was obtained in Paper I. We restrict ourselves to neutrino transparent neutron star cores ( $T \lesssim 10^{10} \mathrm{~K}$ if direct Urca processes are forbidden). We will focus on the viscosity which describes damping of stellar pulsations with frequency $\omega \sim 10^{3}-10^{4} \mathrm{~s}^{-1}$. The pulsation frequency is typically much higher than the rates of Urca processes. In this high-frequency limit, $\zeta$ can be written as a sum of the partial bulk viscosities associated with all Urca processes. For the modified Urca processes $(\mathrm{Nl})$ in $n p e \mu$ matter,

$\zeta=\zeta_{n e}+\zeta_{p e}+\zeta_{n \mu}+\zeta_{p \mu}, \quad \zeta_{N l}=\frac{\left|\lambda_{N l}\right|}{\omega^{2}}\left|\frac{\partial P}{\partial X_{l}}\right| \frac{\partial \eta_{l}}{\partial n_{b}}$

where $\zeta_{N l}$ is a partial bulk viscosity, $P$ is the pressure, $X_{l}=n_{l} / n_{b}$ is an electron or muon fraction $\left(n_{l}\right.$ and $n_{b}=$ $n_{n}+n_{p}$ being the lepton and baryon number densities, respectively), $\eta_{l}=\mu_{n}-\mu_{p}-\mu_{l}, \mu_{j}$ is the chemical potential of particle species $j$, and $\lambda_{N l}$ determines the difference of the rates of the direct and inverse reactions of an Urca process, Eq. (2): $\Gamma_{N l}-\bar{\Gamma}_{N l}=-\lambda_{N l} \eta_{l}$. As in Paper I, we assume that in the absence of pulsations the matter is in the state of chemical equilibrium [with respect to the beta and muon reactions (2)], in which $\eta_{l}=0$. The pulsations are supposed to violate this state only slightly, $|\eta| \ll T$. The derivatives in Eq. (3) are taken at chemical equilibrium with $n_{b}, X_{e}$ and $X_{\mu}$ as independent variables. In our notations, $\partial P / \partial X_{l}$ and $\lambda_{N l}$ are negative.

As shown in Paper I, a partial bulk viscosity can be rewritten as

$\zeta_{N l}=\frac{\left|\lambda_{N l}\right|}{\omega^{2}} C_{l}^{2}, \quad C_{l}=n_{b} \frac{\partial \eta_{l}}{\partial n_{b}}$.
In Paper I the factors $C_{e}$ and $C_{\mu}$ were calculated and fitted by simple formulae for two model equations of state of the npe $\mu$ matter proposed by Prakash et al. (1988). These equations of state correspond to two different forms of the symmetry energy of nucleon matter, $S\left(n_{b}\right)=\left\{13\left[u^{2 / 3}-F(u)\right]+30 F(u)\right\} \mathrm{MeV}$, where $u=n_{b} / n_{0}$ and $n_{0}=0.16 \mathrm{fm}^{-3}$ is the saturation nucleon number density. One has $F(u)=u$ for model I; and $F(u)=2 u^{2} /(1+u)$ for model II.

Thus the problem is reduced to evaluation of the reaction rates $\Gamma_{N l}$ and the factors $\lambda_{N l}$. For a modified Urca process $N l\left(\hbar=c=k_{\mathrm{B}}=1\right)$

$$
\begin{aligned}
\Gamma_{N l}= & \int\left[\prod_{i=1}^{4} \frac{\mathrm{d} \boldsymbol{p}_{i}}{(2 \pi)^{3}}\right] \frac{\mathrm{d} \boldsymbol{p}_{l}}{2 \varepsilon_{l}(2 \pi)^{3}} \frac{\mathrm{d} \boldsymbol{p}_{\nu}}{2 \varepsilon_{\nu}(2 \pi)^{3}} \\
& \times\left(1-f_{N^{\prime}}\right)\left(1-f_{p}\right)\left(1-f_{l}\right) f_{n} f_{N}(2 \pi)^{4} \\
& \times \delta\left(E_{f}-E_{i}\right) \delta\left(\boldsymbol{P}_{f}-\boldsymbol{P}_{i}\right) \frac{1}{s} \sum_{\text {spins }}|M|^{2}
\end{aligned}
$$

where $\boldsymbol{p}_{i}$ is the nucleon momentum $\left(i=n, p, N, N^{\prime}\right), \boldsymbol{p}_{l}$ and $\varepsilon_{l}$ are the electron or muon momentum and energy, while $\boldsymbol{p}_{\nu}$ and $\varepsilon_{\nu}$ are the neutrino momentum and energy. The delta functions $\delta\left(E_{f}-E_{i}\right)$ and $\delta\left(\boldsymbol{P}_{f}-\boldsymbol{P}_{i}\right)$ describe conservation of the energy $E$ and the momentum $\boldsymbol{P}$ of the particles in initial and final states, $i$ and $f$; $|M|^{2}$ is the squared matrix element of the reaction, $s=2$ is the symmetry factor which excludes double counting of the same collisions of identical particles, and $f_{i}=$ $\left\{1+\exp \left[\left(\varepsilon_{i}-\mu_{i}\right) / T\right]\right\}^{-1}$ is the Fermi-Dirac function.

To proceed further we need the matrix element $M$ which depends on the model of nucleon-nucleon interaction. We employ $M$ calculated by Friman \& Maxwell (1979) using the one-pion-exchange model to describe the long-range part of the $N N$ interaction and the Landau Fermi-liquid theory to describe the short-range part. Their result (their Eq. (39)) is that the squared matrix element summed over spin states of all particles and averaged over orientations of the emitted neutrinos can be written in the form:

$$
\sum_{\text {spins }}|M|^{2}=256 G_{\mathrm{F}}^{2} g_{A}^{2}\left(\frac{f^{\pi}}{m_{\pi}}\right)^{4} \frac{\varepsilon_{\nu}}{\varepsilon_{l}} \alpha_{N} \beta_{N} .
$$

Here, $G_{\mathrm{F}}=1.436 \times 10^{-49} \mathrm{erg} \mathrm{\textrm {cm } ^ { 3 }}$ is the Fermi weak coupling constant, $g_{A}=1.26$ is the axial-vector constant of weak hadron current, $f^{\pi} \approx 1$ is the $\pi N$-interaction constant in the $p$-state in the one-pion-exchange model potential, and $m_{\pi}$ is the pion mass $\left(\pi^{ \pm}\right)$. Furthermore, $\alpha_{N}$ describes momentum dependence of the squared matrix element in the Born approximation and $\beta_{N}$ contains various corrections. In principle, these factors are different for the neutron and proton branches of the modified Urca process $\left(\right.$ since $n_{n} \neq n_{p}$ ). Nevertheless, bearing in mind the uncertainties of the employed model, we set $\alpha_{n}=\alpha_{p}$ and $\beta_{n}=\beta_{p}$. Friman \& Maxwell (1979) adopted the values $\alpha_{n}=1.13$ (calculated at $\rho=\rho_{0}$ ) and $\beta_{n}=0.68$ (to account for correlation effects). In our approximation the 
squared matrix element in Eq. (5) can be regarded as constant and can be taken out of the integral.

Further evaluation of $\Gamma_{N l}$ is standard (e.g., Shapiro \& Teukolsky 1983). It is based on phase-space decomposition, neglecting neutrino momentum in the momentumconserving delta function and replacing the momenta of other particles $p_{i}$ by their Fermi momenta $p_{\mathrm{F} i}(i=$ $n, p, l)$ whenever possible. Introducing the dimensionless quantities

$x_{i}=\frac{\varepsilon_{i}-\mu_{i}}{T}, \quad x_{\nu}=\frac{\varepsilon_{\nu}}{T}, \quad \xi=\frac{\eta_{l}}{T}$,

we can present the reaction rate in the form

$$
\begin{aligned}
\Gamma_{N l}= & \Gamma_{N l}^{(0)} I \\
I= & \int \mathrm{d} x_{\nu} x_{\nu}^{2} \int \mathrm{d} x_{n} \mathrm{~d} x_{p} \mathrm{~d} x_{N} \mathrm{~d} x_{N^{\prime}} \mathrm{d} x_{l} \\
& \times f\left(x_{n}\right) f\left(x_{p}\right) f\left(x_{N}\right) f\left(x_{N^{\prime}}\right) f\left(x_{l}\right) \\
& \times \delta\left(x_{n}+x_{p}+x_{N}+x_{N^{\prime}}+x_{l}-x_{\nu}+\xi\right),
\end{aligned}
$$

where $\Gamma_{N l}^{(0)}$ is some typical reaction rate. We have transformed all the blocking factors $(1-f(x))$ into the FermiDirac functions $f(x)$ by replacing $x \rightarrow-x$. The dimensionless energy integral $I$ for the $n$ and $p$ branches of the process is the same. For the reactions involving electrons, we have

$$
\begin{aligned}
\Gamma_{n e}^{(0)} & =\frac{G_{\mathrm{F}}^{2} g_{A}^{2} m_{n}^{* 3} m_{p}^{*} p_{\mathrm{F} p}}{\pi^{9} \hbar^{10} c^{8}}\left(\frac{f^{\pi}}{m_{\pi}}\right)^{4}\left(k_{\mathrm{B}} T\right)^{7} \alpha_{n} \beta_{n} \\
& \approx 3.4410^{25}\left(\frac{m_{n}^{*}}{m_{n}}\right)^{3} \frac{m_{p}^{*}}{m_{p}}\left(\frac{n_{p}}{n_{0}}\right)^{1 / 3} T_{9}^{7} \frac{1}{\mathrm{~cm}^{3} \mathrm{~s}} \\
\Gamma_{p e}^{(0)} & =\Gamma_{n e}^{(0)}\left(\frac{m_{p}^{*}}{m_{n}^{*}}\right)^{2} \frac{\left(3 p_{\mathrm{F} p}+p_{\mathrm{F} e}-p_{\mathrm{F} n}\right)^{2}}{8 p_{\mathrm{F} p} p_{\mathrm{F} e}} \Theta_{p e} .
\end{aligned}
$$

Here, $m_{n}^{*}$ and $m_{p}^{*}$ are the neutron and proton effective masses, respectively, and the step function $\Theta_{p e}$ takes into account the threshold character of the proton reaction branch: it is allowed $\left(\Theta_{p e}=1\right)$ for $p_{\mathrm{F} n}<\left(3 p_{\mathrm{F} p}+p_{\mathrm{Fe} e}\right)$ and forbidden $\left(\Theta_{p e}=0\right)$ otherwise (Yakovlev \& Levenfish 1995). The latter condition holds almost everywhere in the core; it may break at $\rho \lesssim \rho_{0}$ only for the equations of state with small symmetry energy. Notice that Eqs. (10) and (11) have to be slightly modified if the direct Urca process is allowed (due to modification of the angular integrals; e.g., Yakovlev \& Levenfish 1995). However these modifications are of no practical interest: if allowed, the direct Urca process fully dominates over the modified Urca processes.

The rates of the neutron and proton branches of the muon modified Urca process are similar. They are obtained from Eqs. (8) - (11) in two steps. First, by introducing the extra factor $v_{\mathrm{F} \mu} / c=\left(n_{\mu} / n_{e}\right)^{1 / 3}$, where $v_{\mathrm{F} \mu}$ is the Fermi velocity of muons. Second, by replacing $\Theta_{p e} \rightarrow \Theta_{p \mu}$ that corresponds to the replacement $p_{\mathrm{Fe}} \rightarrow p_{\mathrm{F} \mu}$. The muon step function $\Theta_{p \mu}$ allows the proton branch of the process at $p_{\mathrm{F} n}<\left(3 p_{\mathrm{F} p}+p_{\mathrm{F} \mu}\right)$.

The rate $\bar{\Gamma}_{N l}$ of an inverse modified Urca reaction (lepton capture) differs from the rate of the direct reaction $\Gamma_{N l}$ only by the argument of delta function in the expression for $I$; one should replace $\xi \rightarrow-\xi$ there. The difference of the direct and inverse reaction rates can be written as

$$
\begin{aligned}
\Gamma_{N l} & -\bar{\Gamma}_{N l}=-\lambda_{N l} \eta_{l}=\Gamma_{N l}^{(0)} \Delta I, \\
\Delta I & =\int_{0}^{\infty} \mathrm{d} x_{\nu} x_{\nu}^{2}\left[J\left(x_{\nu}-\xi\right)-J\left(x_{\nu}+\xi\right)\right], \\
J(x) & =\int \mathrm{d} x_{n} \mathrm{~d} x_{p} \mathrm{~d} x_{N} \mathrm{~d} x_{N^{\prime}} \mathrm{d} x_{l} f\left(x_{n}\right) f\left(x_{p}\right) f\left(x_{N}\right) \\
& \times f\left(x_{N^{\prime}}\right) f\left(x_{l}\right) \delta\left(x_{n}+x_{p}+x_{N}+x_{N^{\prime}}+x_{l}-x\right) .
\end{aligned}
$$

The function $J(x)$ is evaluated analytically (e.g., Shapiro \& Teukolsky 1983):

$J(x)=\frac{x^{4}+10 \pi^{2} x^{2}+9 \pi^{4}}{24\left(\mathrm{e}^{x}+1\right)}$.

Thus, the difference $\Gamma_{N l}-\bar{\Gamma}_{N l}$ in nonsuperfluid matter is determined by the only parameter $\xi=\eta_{l} / T$. Furthermore, the integral $\Delta I$ is taken analytically:

$$
\begin{aligned}
\Delta I & =\frac{367 \pi^{6}}{1512} \xi \mathcal{F}(\xi), \\
\mathcal{F}(\xi) & =1+\frac{189 \xi^{2}}{367 \pi^{2}}+\frac{21 \xi^{4}}{367 \pi^{4}}+\frac{3 \xi^{6}}{1835 \pi^{6}} .
\end{aligned}
$$

Combined with Eq. (12), this relation gives:

$\left|\lambda_{N l}\right|=\frac{\Gamma_{N l}^{(0)}}{T} \frac{\Delta I}{\xi}$.

As mentioned above, we do not consider large deviations from the chemical equilibrium and restrict ourselves to the case $\left|\eta_{l}\right| \ll T$ in which $\mathcal{F} \approx 1$. It is instructive to estimate characteristic times of Urca processes. Taking into account that $\dot{n}_{e}=\bar{\Gamma}_{N e}-\Gamma_{N e}=\lambda \eta$, we can introduce, for instance, the time $\tau_{w} \sim \delta n_{e} /\left(\lambda_{e} T\right)$ required for an electron Urca process to shift the chemical potential difference by $\eta \sim T$, within the thermal width of the Fermi levels. In this case the electron number density will be changed by $\delta n_{e} \sim n_{e}\left(T / \mu_{e}\right)$. Adopting typical parameters of neutron star matter we have $\tau_{w} \sim 20 T_{9}^{-4}$ s for direct Urca process (Paper I) and $\tau_{w} \sim T_{9}^{-6}$ months for modified Urca processes. Therefore, in any case $\tau_{w}$ is much longer than typical periods of neutron star pulsations $\left(\lesssim 10^{-3} \mathrm{~s}\right)$ which justifies validity of the high-frequency approximation used in our analysis (see above).

Finally, combining Eqs. (4) and (17) we obtain the partial bulk viscosity of the $n p e \mu$ matter, $\zeta_{N l}=\zeta_{N l 0}$ (the subscript " 0 " refers to nonsuperfluid case), produced by the modified Urca processes for $\left|\eta_{l}\right| / T \ll 1$. In standard physical units

$$
\begin{aligned}
& \zeta_{n e 0}=\frac{367 G_{\mathrm{F}}^{2} g_{A}^{2} m_{n}^{* 3} m_{p}^{*} p_{\mathrm{F} p} C_{e}^{2}}{1512 \pi^{3} \hbar^{10} c^{8} \omega^{2}}\left(\frac{f^{\pi}}{m_{\pi}}\right)^{4}\left(k_{\mathrm{B}} T\right)^{6} \alpha_{n} \beta_{n} \\
& \approx 1.4910^{19}\left(\frac{m_{n}^{*}}{m_{n}}\right)^{3} \frac{m_{p}^{*}}{m_{p}}\left(\frac{n_{p}}{n_{0}}\right)^{1 / 3}\left(\frac{C_{e}}{100 \mathrm{MeV}}\right)^{2} \\
& \times T_{9}^{6} \omega_{4}^{-2} \alpha_{n} \beta_{n} \quad \mathrm{~g} \mathrm{~cm}^{-1} \mathrm{~s}^{-1}, \\
& \zeta_{p e 0}=\zeta_{n e 0}\left(\frac{m_{p}^{*}}{m_{n}^{*}}\right)^{2} \frac{\left(3 p_{\mathrm{F} p}+p_{\mathrm{F} e}-p_{\mathrm{F} n}\right)^{2}}{8 p_{\mathrm{F} p} p_{\mathrm{F} e}} \Theta_{p e}
\end{aligned}
$$


$\zeta_{n \mu 0}=\zeta_{n e 0}\left(\frac{p_{\mathrm{F} \mu}}{p_{\mathrm{Fe}}}\right)\left(\frac{C_{\mu}}{C_{e}}\right)^{2}$

$\zeta_{p \mu 0}=\zeta_{n e 0}\left(\frac{C_{\mu} m_{p}^{*}}{C_{e} m_{n}^{*}}\right)^{2} \frac{\left(3 p_{\mathrm{F} p}+p_{\mathrm{F} \mu}-p_{\mathrm{F} n}\right)^{2}}{8 p_{\mathrm{F} p} p_{\mathrm{F} \mu}}\left(\frac{p_{\mathrm{F} \mu}}{p_{\mathrm{F} e}}\right) \Theta_{p \mu}$,

where $\omega_{4}=\omega /\left(10^{4} \mathrm{~s}^{-1}\right)$ and $T_{9}=T /\left(10^{9} \mathrm{~K}\right)$.

For the two equations of state I and II (see above), the density dependence of the partial bulk viscosities is fitted by simple expressions presented in the Appendix. Figure 1 shows the total bulk viscosity of nonsuperfluid matter versus the baryon number density $n_{b}$ for both models. The contributions of the modified and direct Urca processes are included; the latter contribution is calculated using the results of Paper I. The dotted curves show the bulk viscosity for the simplified equations of state based on the same nuclear energy but with the appearance of muons artificially forbidden.

The viscosities for models I and II are similar. The muon thresholds are $n_{b}=0.150 \mathrm{fm}^{-3}$ and $0.152 \mathrm{fm}^{-3}$ for models I and II. At lower densities the muons are absent and the bulk viscosity is produced by the electron modified Urca processes (and the dotted curves are identical to the solid ones). At densities above the thresholds the muon modified Urca processes are switched on and amplify the bulk viscosity. Since $\zeta_{N \mu} \propto v_{\mathrm{F} \mu}$, the muon bulk viscosity is switched on without any jump. On the contrary, the bulk viscosity due to the direct Urca processes is switched on in a jump-like manner at their threshold densities. The thresholds for the electron direct Urca process are $n_{b}=0.414 \mathrm{fm}^{-3}$ and $0.302 \mathrm{fm}^{-3}$, for models I and II. As explained in Paper I, the presence of muons lowers the threshold density, mainly due to increasing the number density and Fermi momenta of protons. On the other hand, the muons lower the bulk viscosity produced by the electron direct Urca processes (by decreasing $n_{e}$ ). At larger $n_{b}$ the total bulk viscosity jumps again, at $n_{b}=0.503 \mathrm{fm}^{-3}$ and $0.358 \mathrm{fm}^{-3}$ for models I and II. This time the jump is associated with switching on the muon direct Urca process. The contribution of the muon direct Urca into the bulk viscosity is even larger than the contribution of the electron direct Urca (Paper I). With this contribution the bulk viscosity becomes larger than in the npe matter.

\section{Bulk viscosity of superfluid matter}

\subsection{Superfluid reduction factors}

Now we turn to superfluid reduction of the bulk viscosity produced by the modified Urca processes. As in Paper I, we restrict ourselves to the motions of the $n p e \mu$ fluid in which all components move with same macroscopic velocity. This assumption reduces equations of stellar pulsations to those of one-fluid hydrodynamics, with a single coefficient of the bulk viscosity; our bulk viscosity $\zeta$ is identical to $\zeta_{2}$ of Landau \& Lifshitz (1987). The superfluid reduction of the bulk viscosity is similar to the superfluid reduction of the neutrino emissivity. Since the

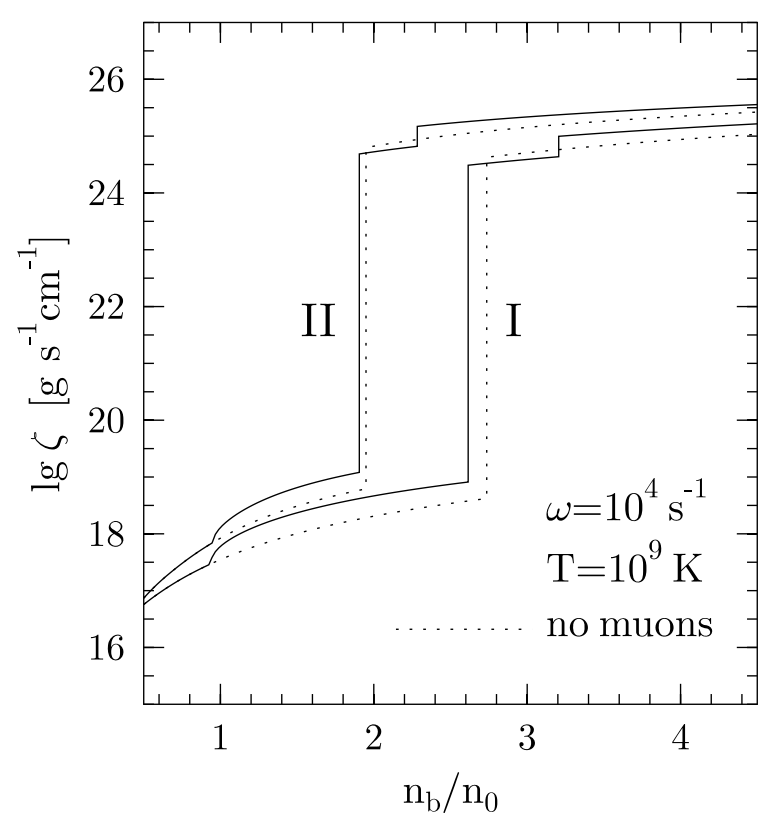

Fig. 1. The bulk viscosity $\zeta$ for models I and II of nonsuperfluid npe $\mu$ matter (solid lines), produced by direct and modified Urca processes involving electrons and muons, versus the baryon number density $n_{b}$, for $T=10^{9} \mathrm{~K}$ and $\omega=10^{4} \mathrm{~s}^{-1}$. Dotted lines show the same bulk viscosity but for the models of matter without muons. Jumps of the curves are associated with opening direct Urca processes (see the text).

latter problem has been the subject of extensive studies (e.g., Yakovlev et al. 1999) we omit technical details.

We adopt the traditional assumption (e.g., Yakovlev et al. 1999) that the protons form Cooper pairs due to singlet-state $\left({ }^{1} \mathrm{~S}_{0}\right)$ pairing (superfluidity of type $\mathrm{A}$, in notations of Paper I). As for the neutrons, they will be thought to undergo either the singlet-state pairing or the triplet-state $\left({ }^{3} \mathrm{P}_{2}\right)$ pairing with zero projection of the total angular momentum of the Cooper pair on the quantization axis (superfluidity of type B). Numerous microscopic calculations (see Yakovlev et al. 1999 for references) show that the singlet-state pairing of neutrons takes place at $\rho \lesssim \rho_{0}$, while their triplet-state pairing is realized at higher densities. The critical temperatures $T_{c n}$ and $T_{c p}$ of the neutron and proton superfluids are very model dependent, and we treat them as free parameters.

Microscopically, superfluidity introduces a gap $\delta$ into momentum dependence of the nucleon energy, $\varepsilon(\boldsymbol{p})$. Near the Fermi surface $\left(\left|p-p_{\mathrm{F}}\right| \ll p_{\mathrm{F}}\right)$, this dependence can be written as

$$
\begin{array}{ll}
\varepsilon=\mu-\sqrt{\delta^{2}+v_{\mathrm{F}}^{2}\left(p-p_{\mathrm{F}}\right)^{2}} & \text { at } p<p_{\mathrm{F}}, \\
\varepsilon=\mu+\sqrt{\delta^{2}+v_{\mathrm{F}}^{2}\left(p-p_{\mathrm{F}}\right)^{2}} & \text { at } p \geq p_{\mathrm{F}} .
\end{array}
$$

In case $\mathrm{A}$ the gap is isotropic (independent of orientation of $\boldsymbol{p}), \delta_{\mathrm{A}}=\Delta_{\mathrm{A}}(T)$, while in case B the gap depends on the angle $\vartheta$ between $\boldsymbol{p}$ and the quantization axis, $\delta_{\mathrm{B}}=\Delta_{\mathrm{B}}(T)\left(1+3 \cos ^{2} \vartheta\right)^{1 / 2}$. In both cases $\Delta(T)$ is the amplitude which describes temperature dependence of the 
gap. The amplitude is derived from the standard equation of the Bardeen-Cooper-Schrieffer theory.

For further analysis we introduce the dimensionless quantities $\left(k_{\mathrm{B}}=1\right.$ as before):

$v=\frac{\Delta(T)}{T}, \quad \tau=\frac{T}{T_{c}}, \quad y=\frac{\delta}{T}$.

The dimensionless gap $y$ can be presented in the form: $y_{\mathrm{A}}=v_{\mathrm{A}}, y_{\mathrm{B}}=v_{\mathrm{B}} \sqrt{1+3 \cos ^{2} \vartheta}$. The dimensionless gap amplitude $v$ depends only on $\tau=T / T_{c}$. This dependence is accurately fitted as (Levenfish \& Yakovlev 1994):

$v_{\mathrm{A}}=\sqrt{1-\tau}(1.456-0.157 / \sqrt{\tau}+1.764 / \tau)$,

$v_{\mathrm{B}}=\sqrt{1-\tau}(0.7893+1.188 / \tau)$.

Quite generally, the high-frequency bulk viscosity can be presented in the form

$\zeta=\sum_{N l} \zeta_{N l 0} R^{(N)}$

where $\zeta_{N l 0}$ is a partial bulk viscosity of nonsuperfluid matter (given by Eqs. (18)-(21)) and $R^{(N)}=R_{N l}^{(N)}$ is a factor which describes its superfluid reduction. The factors for the modified Urca processes $N e$ and $N \mu$ are equal since the superfluidity affects only nucleons. In non-superfluid matter, we have $R^{(N)}=1$ and reproduce the results of Sect. 2.

Thus the problem consists in calculating the reduction factors $R^{(N)}$. Each factor depends on two parameters, $v_{n}$ and $v_{p}$, dimensionless gap amplitudes of the neutrons and protons. As in Paper I we may assume that the superfluidity affects noticeably only the factor $\lambda_{N l}$ in the expression for the bulk viscosity, Eq. (4). By making the standard angular-energy decomposition in the expressions for the reaction rates $\Gamma_{N l}$ and $\bar{\Gamma}_{N l}$, one can easily show that $\lambda_{N l}$ contains the factor

$\mathcal{J}\left(v_{p}, v_{n}\right)=4 \pi \int\left[\prod_{i=1}^{5} \mathrm{~d} \Omega_{i}\right] \delta\left(\sum_{i=1}^{5} \boldsymbol{p}_{i}\right) \Delta I$,

where $\mathrm{d} \Omega_{i}$ is the solid angle element in the direction of $\boldsymbol{p}_{i}$ and $\Delta I$ is the energy integral given by Eq. (13). This integral is the main quantity affected by the superfluidity. At $\xi \ll 1$ the integrand of Eq. (13) contains the function $J\left(x_{\nu}-\xi\right)-J\left(x_{\nu}+\xi\right) \approx-2 \xi \partial J\left(x_{\nu}\right) / \partial x_{\nu}$, where $J\left(x_{\nu}\right)$ is given by Eq. (14). Thus, for small deviations from equilibrium one can transform Eq. (13) to:

$$
\begin{aligned}
\Delta I= & 4 \xi \int_{0}^{+\infty} \mathrm{d} x_{\nu} x_{\nu}\left[\prod_{i=1}^{5} \int_{-\infty}^{+\infty} \mathrm{d} x_{i} f\left(x_{i}\right)\right] \\
& \times \delta\left(\sum_{i=1}^{5} x_{i}-x_{\nu}\right)
\end{aligned}
$$

Here, $i=n, p, N, N^{\prime}, l$.

Let us now label the quantities in the nonsuperfluid case by the subscript " 0 ". The angular-energy decomposition yields

$\mathcal{J}_{0}=A_{n l 0} \Delta I_{0}, \quad \Delta I_{0}=\frac{367 \pi^{6} \xi}{1512}$.
In this case $A_{n l 0}$ is the angular integral containing the momentum conserving delta function, in which the neutrino momentum is neglected and the momenta of all other particles are placed at their Fermi surfaces. For the processes of our study:

$$
\begin{aligned}
& A_{n l 0}=\frac{(4 \pi)^{5}}{2 p_{\mathrm{F} n}^{3}} \\
& A_{p l 0}=\frac{(4 \pi)^{5}}{2 p_{\mathrm{F} n}^{2} p_{\mathrm{F} p}} \frac{\left(3 p_{\mathrm{F} p}+p_{\mathrm{F} l}-p_{\mathrm{F} n}\right)^{2}}{8 p_{\mathrm{F} p} p_{\mathrm{F} l}} \Theta_{p l} .
\end{aligned}
$$

Notice that Yakovlev \& Levenfish (1995) inaccurately determined $A_{p l 0}$. Here we present the corrected expression.

Generalization of $\lambda_{N l}$ to the superfluid case can be achieved by introducing the neutron and proton energy gaps into Eq. (26). Then

$\lambda_{N l}=\lambda_{N l 0} R^{(N)}, \quad R^{(N)}\left(v_{p}, v_{n}\right)=\frac{\mathcal{J}}{A_{N l 0} \Delta I_{0}}$,

where $\mathcal{J}$ is given by Eq. (26) with

$$
\begin{aligned}
\Delta I\left(y_{1}, y_{2}\right)= & 4 \xi \int_{0}^{+\infty} \mathrm{d} x_{\nu} x_{\nu}\left[\prod_{i=1}^{5} \int_{-\infty}^{+\infty} \mathrm{d} x_{i} f\left(z_{i}\right)\right] \\
& \times \delta\left(\sum_{i=1}^{5} z_{i}-x_{\nu}\right)
\end{aligned}
$$

$z_{i}=\left(\varepsilon_{i}-\mu\right) / T=\operatorname{sign}\left(x_{i}\right) \sqrt{x_{i}^{2}+y_{i}^{2}}$ for nucleons, and $z_{l}=$ $x_{l}$. Equation (30) is the general expression for calculating the reduction factor $R^{(N)}$.

\subsection{Superfluidity of neutrons or protons}

First, let the neutrons be normal while the protons undergo Cooper pairing of type A. Since the energy gap is isotropic, the angular integral in Eq. (26) is separated from the energy one, being the same as in the nonsuperfluid case, Eq. (29). Then in Eq. (26) we can put $y_{n}=0$ and $y_{p}=v_{p}$. Accordingly, the reduction factor depends on the only parameter $v=v_{p}$. For a strong proton superfluidity $\left(\tau=T / T_{c p} \ll 1, v \gg 1\right)$, the asymptotes of the reduction factors for the neutron and proton branches of the modified Urca process are:

$$
\begin{aligned}
R_{p \mathrm{~A}}^{(n)} & =\frac{126 \sqrt{2}}{1835 \pi^{5.5}} v^{5.5} \mathrm{e}^{-v}=\frac{0.004061}{\tau^{5.5}} \exp \left(-\frac{1.764}{\tau}\right) \\
R_{p \mathrm{~A}}^{(p)} & =\frac{6048}{367 \pi^{6}} \gamma v^{5} \mathrm{e}^{-2 v}=\frac{0.1604}{\tau^{5}} \exp \left(-\frac{3.528}{\tau}\right) \\
\gamma & =\frac{\pi}{16}\left(21 \sqrt{3}-\frac{51}{2} \ln (\sqrt{3}+2)\right) \approx 0.548
\end{aligned}
$$

The proton branch is affected by the proton superfluidity stronger than the neutron branch. This is natural (e.g., Yakovlev et al. 1999) because in the proton branch we have three protons which belong to the superfluid component of matter, while in the neutron branch there is only one such particle. Under the number of reacting nucleons we mean their total number (in the initial plus final states) in the direct or inverse reaction of an Urca process. 
We have calculated $R_{p \mathrm{~A}}^{(n)}$ and $R_{p \mathrm{~A}}^{(p)}$ numerically in a wide range of $v=v_{p}$ and proposed the fits which reproduce the numerical results (with the mean errors of $\lesssim 1 \%$ ) and the asymptotes (32) and (33):

$$
\begin{aligned}
R_{p \mathrm{~A}}^{(n)}= & \frac{a^{5.5}+b^{3.5}}{2} \exp \left(3.245-\sqrt{(3.245)^{2}+v^{2}}\right), \\
& a=0.1863+\sqrt{(0.8137)^{2}+(0.1310 v)^{2}} \\
b & =0.1863+\sqrt{(0.8137)^{2}+(0.1437 v)^{2}}
\end{aligned}
$$

$$
\begin{array}{r}
R_{p \mathrm{~A}}^{(p)}=c^{5} \exp \left(5.033-\sqrt{(5.033)^{2}+(2 v)^{2}}\right), \\
c=0.3034+\sqrt{(0.6966)^{2}+(0.1437 v)^{2}} .
\end{array}
$$

Now consider normal protons and superfluid neutrons (superfluidity of type B). Then in Eq. (26) we may put $z_{p}=x_{p}$ and the reduction factor depends only on $v=v_{n}$. For a strong neutron superfluidity $\left(\tau=T / T_{c n} \ll 1\right.$, $v \gg 1)$ the asymptotes of the reduction factors are

$$
\begin{aligned}
& R_{n \mathrm{~B}}^{(n)}=\frac{6048}{367 \pi^{6}} \frac{2 \gamma}{3 \sqrt{3}} v^{4} \mathrm{e}^{-2 v}=\frac{0.00720}{\tau^{4}} \exp \left(-\frac{2.376}{\tau}\right), \\
& R_{n \mathrm{~B}}^{(p)}=\frac{42 \sqrt{3}}{1835 \pi^{5}} v^{5} \mathrm{e}^{-v}=\frac{3.06610^{-4}}{\tau^{5}} \exp \left(-\frac{1.188}{\tau}\right) .
\end{aligned}
$$

In this case the neutron branch is affected by the superfluidity stronger than the proton branch.

The calculation of $R_{n \mathrm{~B}}^{(n)}\left(v_{n}\right)$ for intermediate values $v_{n}$ is difficult since there are three superfluid neutrons with anisotropic gaps. An approximate expression for $R_{n \mathrm{~B}}^{(n)}\left(v_{n}\right)$ will be proposed in Sect. 3.3. As for the factor $R_{n \mathrm{~B}}^{(p)}$, we have calculated it numerically for a wide range of $v$ and obtained the fit which reproduces the numerical results (with mean error $\lesssim 1 \%$ ) and the asymptotes:

$$
\begin{aligned}
R_{n \mathrm{~B}}^{(p)}= & \frac{a^{5}+b^{3}}{2} \exp \left(2.110-\sqrt{(2.110)^{2}+v^{2}}\right) \\
& a=0.1973+\sqrt{(0.8027)^{2}+(0.1257 v)^{2}} \\
& b=0.1973+\sqrt{(0.8027)^{2}+(0.1428 v)^{2}}
\end{aligned}
$$

The factors $R^{(N)}$ which describe reduction of the bulk viscosity of npe $\mu$ matter by superfluidity of neutrons or protons are plotted in Figs. 2 and 3. For comparison, we present also the reduction factors $R^{(\mathrm{D})}$ for the partial bulk viscosity produced by the direct Urca processes (Paper I). Figure 2 displays the reduction factors versus $T / T_{c}$, while Fig. 3 presents them versus $v$. As seen from these figures, the reduction factors may be separated into two groups. The first group contains the factors $R_{p \mathrm{~A}}^{(n)}, R_{n \mathrm{~B}}^{(p)}, R_{p \mathrm{~A}}^{(\mathrm{D})}, R_{n \mathrm{~B}}^{(\mathrm{D})}$ which describe reduction of the reactions with one superfluid nucleon. The other group contains the factors $R_{p \mathrm{~A}}^{(p)}$ and $R_{n \mathrm{~B}}^{(n)}$ (asymptote) for the reactions with three superfluid nucleons at once. Notice that the curves inside each group in Fig. 3 are much closer to one another than in Fig. 2. The same situation takes place for the reduction factors of neutrino emissivities (e.g., Yakovlev et al. 1999).

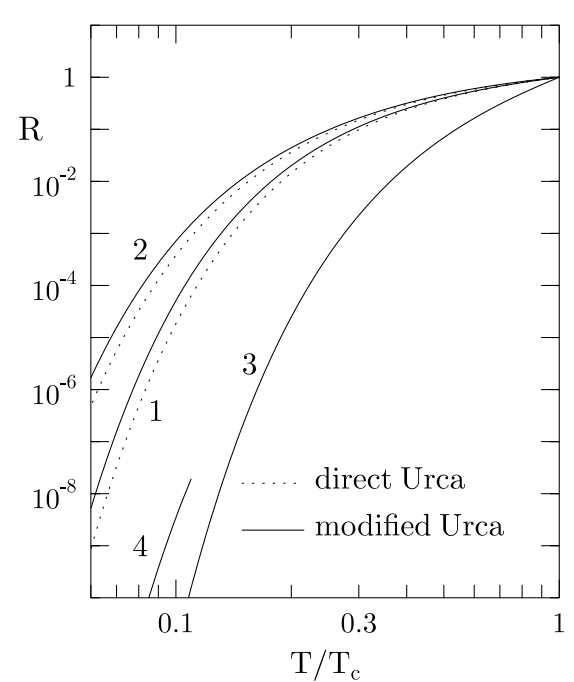

Fig. 2. Factors $R$ which describe reduction of partial bulk viscosities of npe $\mu$ matter by superfluidity of neutrons or protons versus $T / T_{c}$. Solid and dotted lines refer to the modified and direct Urca processes, respectively. Curves 1 are for the viscosities due to the neutron modified Urca and the direct Urca processes and the proton superfluidity. Curves 2 are for the proton modified Urca and the direct Urca viscosities and the neutron superfluidity. Curve 3 is for the proton modified Urca process and the neutron superfluidity. Curve 4 is the asymptote for the neutron modified Urca and the neutron superfluidity.

\subsection{Superfluidity of neutrons and protons}

If the neutrons and protons are superfluid at once, calculation of the reduction factors is complicated. If however we do not need very accurate results, we can use approximate similarity relations analogous to those suggested by Yakovlev \& Levenfish (1995) and described also by Yakovlev et al. (1999) for the reduction of neutrino emissivities in different reactions:

$R_{\mathrm{AB}}^{(p)}\left(v_{p}, v_{n}\right) \approx \frac{R_{\mathrm{AB}}^{(\mathrm{D})}\left(2 v_{p}, v_{n}\right)}{R_{n \mathrm{~B}}^{(\mathrm{D})}\left(v_{n}\right)} R_{n \mathrm{~B}}^{(p)}\left(v_{n}\right)$,
$R_{\mathrm{AB}}^{(n)}\left(v_{p}, v_{n}\right) \approx \frac{R_{\mathrm{AB}}^{(\mathrm{D})}\left(v_{p}, 2 v_{n}\right)}{R_{p \mathrm{~A}}^{(\mathrm{D})}\left(v_{p}\right)} R_{p \mathrm{~A}}^{(n)}\left(v_{p}\right)$.

Here, $R_{p \mathrm{~A}}^{(\mathrm{D})}, R_{n \mathrm{~B}}^{(\mathrm{D})}$ and $R_{\mathrm{AB}}^{(\mathrm{D})}$ are the factors which define reduction of the direct-Urca bulk viscosity by superfluidity of protons, neutrons and protons+neutrons, respectively. These factors were obtained in Paper I. Notice that Eq. (39) becomes exact for normal protons $\left(v_{p}=0\right)$, while Eq. (40) is exact for normal neutrons. We may assume also that the reduction of the $N$-branch of the modified-Urca viscosity by neutron superfluidity can be approximated (for $v \lesssim 10$ ) by the factor: $R_{n \mathrm{~B}}^{(n)} \approx R_{p \mathrm{~A}}^{(p)}\left(v_{n}\right)$.

Finally, Fig. 4 illustrates reduction of the bulk viscosity of $n p e \mu$ matter (due to the modified Urca processes) with decreasing temperature by superfluidity of neutrons of type $\mathrm{B}$ or protons of type $\mathrm{A}$ for $n_{b}=2 n_{0}$ and $\omega=10^{4} \mathrm{~s}^{-1}$. Thick solid line shows the viscosity of non-superfluid matter (cf. with Fig. 1). Thin dashed 


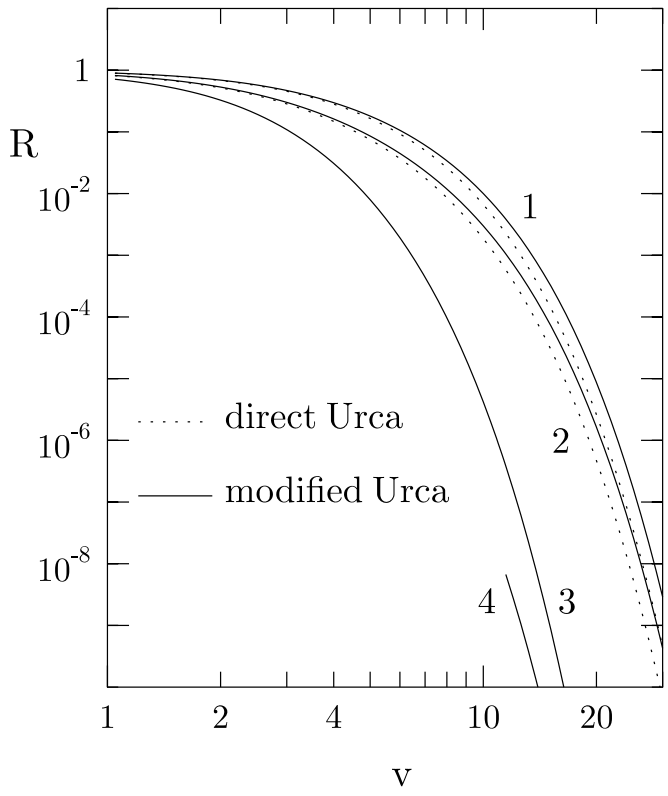

Fig. 3. Same as in Fig. 2 but versus dimensionless energy gap parameter $v$.

lines present the bulk viscosity suppressed by the proton superfluidity at several selected critical temperatures $T_{c p}$ indicated near the curves. The dot-and-dashed line shows the effect of neutron superfluidity $\left(T_{c n}=10^{10} \mathrm{~K}\right)$ for normal protons. We see that superfluid reduction of the bulk viscosity depends on temperature, superfluidity type, and the critical temperatures $T_{c n}$ and $T_{c p}$. If $T_{c n}$ and $T_{c p}$ are not higher than $10^{10} \mathrm{~K}$ for $n_{b} \sim 2 n_{0}$, then the superfluid reduction cannot be very large, say, for $T \gtrsim 10^{9} \mathrm{~K}$. It cannot reach more than two orders of magnitude in the case of superfluidity of one nucleon species or six orders of magnitude if $n$ and $p$ are superfluid at once for $T=10^{9} \mathrm{~K}$ at $T_{c n}=T_{c p}=10^{10} \mathrm{~K}$ (Fig. 5 of Paper I). The reduction grows exponentially with further decrease of $T$.

\section{Conclusions}

We have calculated the bulk viscosity due to nonequilibrium modified Urca processes in neutron star cores composed of neutrons, protons, electrons, and muons $(n p e \mu)$. We have considered non-superfluid matter (Sect. 2) and described also reduction of the bulk viscosity by superfluidity of neutrons and protons (Sect. 3). In combination with our previous work (Paper I) we have obtained realistic description of the bulk viscosity provided by the modified and direct Urca processes in the npe $\mu$ matter of the neutron star cores. The results can be used for studying damping of pulsations in neutron stars and gravitational radiation driven instabilities in rotating neutron stars. Strong superfluidity of neutrons and protons reduces the bulk viscosity and creates favorable conditions for the development of these instabilities.

Acknowledgements. Two of the authors (KPL and DGY) acknowledge hospitality of N. Copernicus Astronomical Center in

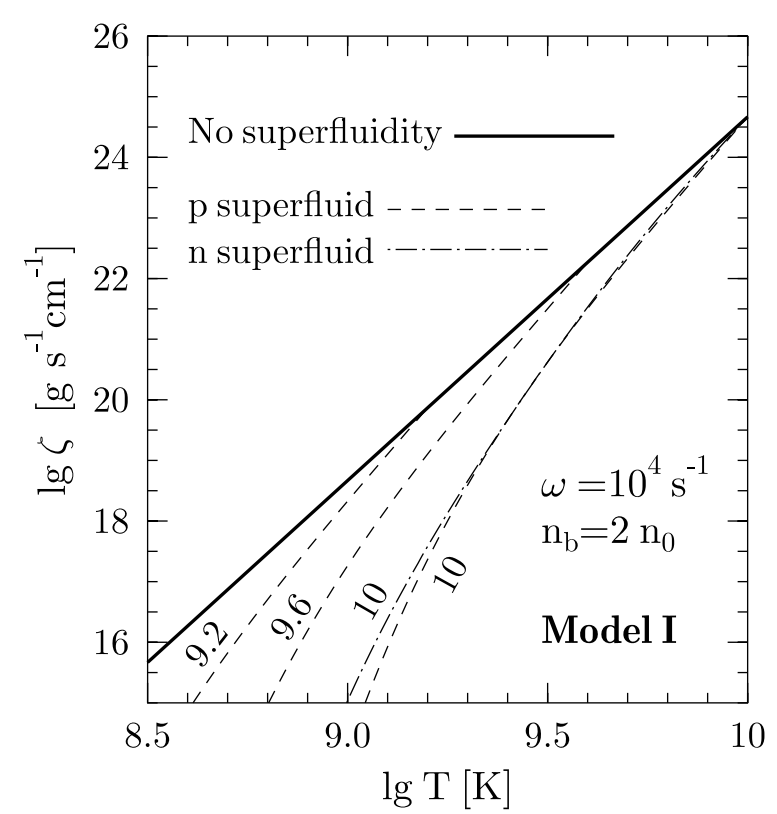

Fig. 4. Bulk viscosity of superfluid npe $\mu$ matter (model I) produced by the electron and muon modified Urca processes at the baryon number density $n_{b}=2 n_{0}$ and $\omega=10^{4} \mathrm{~s}^{-1}$ as a function of $T$. Thick solid line corresponds to non-superfluid matter, dashed lines are for matter with superfluid protons $\left(T_{c p}=10^{10}\right.$, $10^{9.6}$ and $10^{9.2} \mathrm{~K}$ ) and normal neutrons, while dot-and-dashed line is for matter with superfluid neutrons $\left(T_{c n}=10^{10} \mathrm{~K}\right)$ and normal protons. Curves are labeled by $\lg T_{c p}$ or $\lg T_{c n}$.

Warsaw. The authors are grateful to anonymous referee for useful comments. This work was supported in part by the RBRF (grant No. 99-02-18099), INTAS (grant No. 96-0542), and KBN (grant 2 P03D 014 13).

\section{Appendix}

To calculate the bulk viscosity from the equations obtained above one needs to know the factors $C_{e}$ and $C_{\mu}$ and the number densities $n_{n}, n_{p}, n_{e}, n_{\mu}$, and $n_{b}$. Other functions of density such as $\Gamma_{N l}$ are expressed through the Fermi-momenta of various particles $p_{\mathrm{F} i}=\hbar\left(3 \pi^{2} n_{i}\right)^{1 / 3}$ and hence through $n_{i}$. The practical expression for calculating $C_{e}$ and $C_{\mu}$ for any given equation of state was obtained in Paper I (Eq. (19)). The number densities $n_{i}$ are usually available for a given equation of state. It is sufficient to specify $n_{p}, n_{\mu}$ and $n_{b}$, since $n_{n}=n_{b}-n_{p}$ and $n_{e}=n_{p}-n_{\mu}$.

For example, consider two model equations of state I and II used in Paper I and in the present paper. The fits of the quantities $q_{l}=\left(n_{l} / n_{0}\right)^{1 / 3}\left(C_{l} / 100 \mathrm{MeV}\right)^{2}, l=e$ and $\mu$, as functions of the baryon number density $n_{b}$ are given by Eq. (36) of Paper I. They yield practical expressions for $C_{l}\left(n_{b}\right)$ provided the particle number densities are known. Thus, we must fit the number densities $n_{i}=n_{i}\left(n_{b}\right)$. We present the proton number density as $n_{p}=n_{p 0}+\Delta n_{p}$, where $n_{p 0}$ is the proton number density for the case in which creation of muons is artificially forbidden. For the equations of state I and II the muons appear at $n_{b}=n_{b \mu}=0.15000$ and $0.5122831 \mathrm{fm}^{-3}$, respectively. 
Therefore, we have $n_{p}=n_{p 0}=n_{e}$ and $n_{\mu}=0$ for $n_{b} \leq n_{b \mu}$.

We have fitted $n_{p 0}$ in the density range from $n_{b}=0.048$ $\mathrm{fm}^{-3}=0.3 n_{0}$ to $1.92 \mathrm{fm}^{-3}=12 n_{0}$ as

$n_{p 0}=\eta+\alpha n^{\beta} /\left(1+\gamma n^{\delta}\right)$,

and we have also fitted $\Delta n_{p}$ and $n_{\mu}$ in the range from $n_{b}=n_{b \mu}$ to $1.92 \mathrm{fm}^{-3}$ as

$$
\begin{aligned}
\Delta n_{p} & =a \delta n^{1.5}\left(1+d \delta n^{e}\right) /\left(1+b \delta n^{c}\right), \\
n_{\mu} & =A \delta n^{1.5}\left(1+D \delta n^{E}\right) /\left(1+B \delta n^{C}\right) .
\end{aligned}
$$

Here, $n \equiv n_{b}, \delta n=n_{b}-n_{b \mu}$, and all number densities are expressed in $\mathrm{fm}^{-3}$. The fit parameters are $\alpha=0.7295, \beta=2.503, \gamma=2.604, \delta=1.307, \eta=0$, $a=0.1306, b=1.887, c=1.509, A=0.1614, B=0.1785$, $C=1.235, d=D=0$ for model I; $\alpha=3.64, \beta=3.374$, $\gamma=12.07, \delta=2.096, \eta=2.673 \times 10^{-5}, a=0.1919$, $b=4.946, c=1.279, d=0.1558, e=0.9253, A=0.2376$, $B=3.294, C=1.468, D=2.194, E=1.201$ for model II. The maximum errors of the fits of $n_{p}$ and $n_{\mu}$ do not exceed $1.3 \%$ in the indicated density ranges. The presented fits give all functions versus $n_{b}$. The fit expressions of $n_{b}$ via mass density $\rho$ for three versions of each equation of state I and II corresponding to three different values of the compression modulus of saturated nuclear matter, $K=120,180$ and $240 \mathrm{MeV}$, are given by Eq. (37) of Paper I.

\section{References}

Andersson, N., \& Kokkotas, K. D. 2000, Int. J. Mod. Phys. D, in press [arXiv: gr-qc/0010102]

Cutler, C., Lindblom, L., \& Splinter, R. J. 1990, ApJ, 363, 603

Flowers, E., \& Itoh, N. 1976, ApJ, 206, 518

Friman, B. L., \& Maxwell, O. V. 1979, ApJ, 232, 541

Haensel, P., \& Schaeffer, R. 1992, Phys. Rev. D, 45, 4708

Haensel, P., Levenfish, K. P., \& Yakovlev, D. G. 2000, A\&A, 357, 1157, Paper I

Landau, L. D., \& Lifshitz, E. M. 1987, Fluid Mechanics (Pergamon, Oxford)

Lattimer, J. M., Pethick, C. J., Prakash, M., \& Haensel, P. 1991, Phys. Rev. Lett., 66, 2701

Lindblom, L. 1995, ApJ, 438, 265

Levenfish, K. P., \& Yakovlev, D. G. 1994, Astron. Lett., 20, 43

Prakash, M., Ainsworth, T. L., \& Lattimer, J. M. 1988, Phys. Rev. Lett., 61, 2518

Sawyer, R. F. 1989, Phys. Rev. D, 39, 3804

Shapiro, S. L., \& Teukolsky, S. A. 1983, Black Holes White Dwarfs and Neutron Stars (Wiley-Interscience, New-York)

Yakovlev, D. G., \& Levenfish, K. P. 1995, A\&A, 297, 717

Yakovlev, D. G., Levenfish, K. P., \& Shibanov, Yu. A. 1999, Physics-Uspekhi, 42, 737 [arXiv: astro-ph/9906456]

Zdunik, J. L. 1996, A\&A, 308, 828 